\title{
Simulating the structure and texture of solid wood
}

\author{
Albert Julius Liu * \\ Cornell University
}

\author{
Zhao Dong \\ Autodesk, Inc.
}

\author{
Miloš Hašan \\ Autodesk, Inc.
}

Steve Marschner
Cornell University

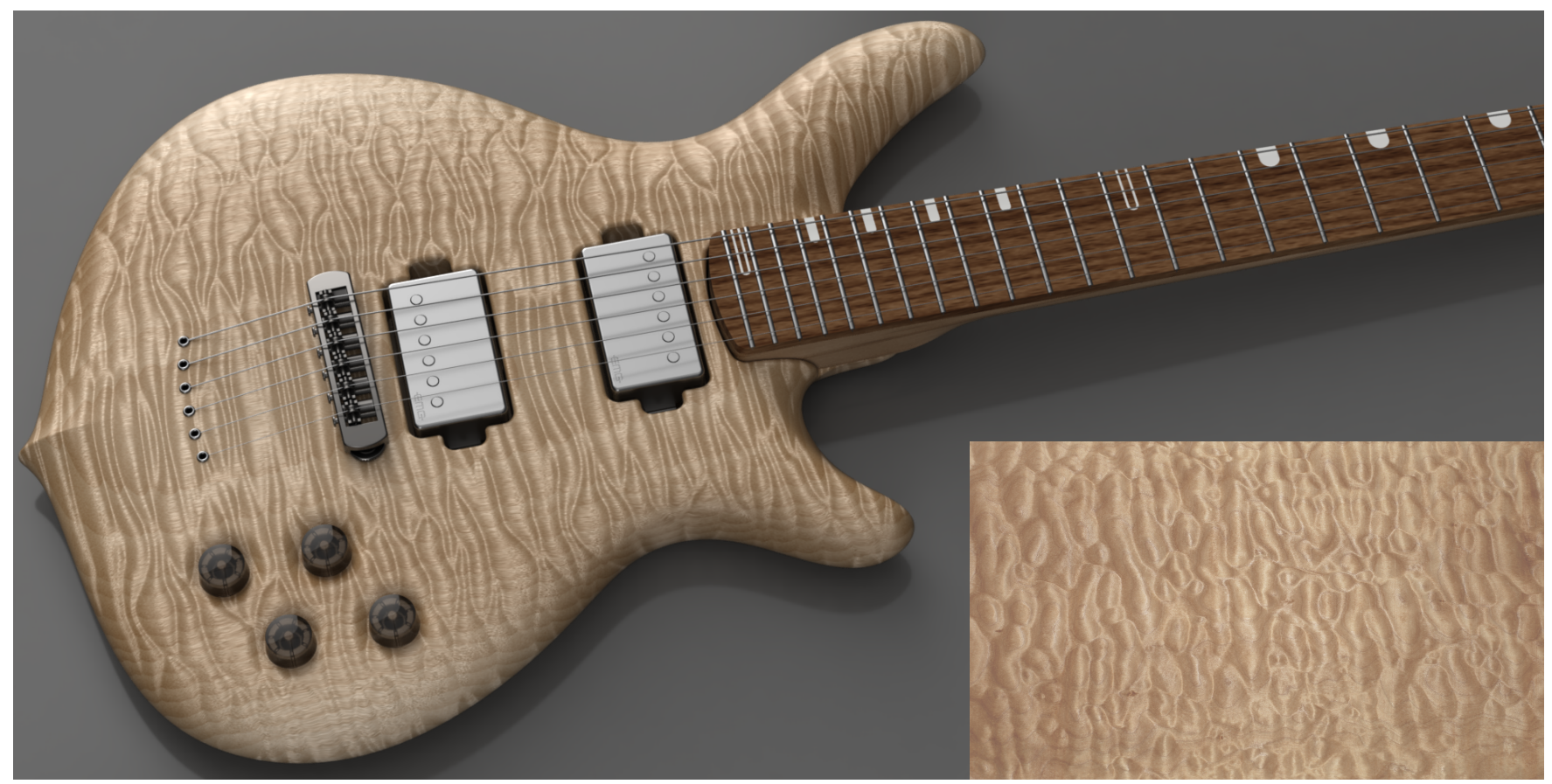

Figure 1: A guitar model featuring a couple different wood materials: quilted maple on the body and walnut on the neck. The image is rendered using our comprehensive, volumetric, procedural model of wood. We simulate most of the significant wood features: growth rings, pores, rays, and growth distortions. Furthermore, our model can produce the anisotropic specular highlight arising from reflection from the subsurface fiber structure, as seen in the quilted maple figure. The fiber directions are automatically derived from the growth distortions. Model by Nikos Natsios. Inset: A photograph of real quilted maple.

\begin{abstract}
Wood is an important decorative material prized for its unique appearance. It is commonly rendered using artistically authored 2D color and bump textures, which reproduces color patterns on flat surfaces well. But the dramatic anisotropic specular figure caused by wood fibers, common in curly maple and other species, is harder to achieve. While suitable BRDF models exist, the texture parameter maps for these wood BRDFs are difficult to author-good results have been shown with elaborate measurements for small flat samples, but these models are not much used in practice. Furthermore, mapping 2D image textures onto 3D objects leads to distortion and inconsistencies. Procedural volumetric textures solve these geometric problems, but existing methods produce much lower quality than image textures. This paper aims to bring the best of all these techniques together: we present a comprehensive volumetric simulation of wood appearance, including growth rings, color variation, pores, rays, and growth distortions. The fiber directions required for anisotropic specular figure follow naturally from the distortions. Our results rival the quality of textures based on photographs, but with the consistency and convenience of a volumetric model. Our model is modular, with components that are intuitive to control, fast to compute, and require minimal storage.
\end{abstract}

Keywords: BRDF, solid texture, texture synthesis, wood

\footnotetext{
*ajul@cs.cornell.edu
}

Concepts: •Computing methodologies $\rightarrow$ Reflectance modeling; Texturing; Volumetric models;

\section{Introduction}

Wood is a common decorative material in our surroundings, particularly in indoor scenes full of wooden floors and furniture, and also used in the design of many products (Figure 1). The conventional way to render wood is to use high-resolution $2 \mathrm{D}$ textures. These are commonly authored by using photographs to control the diffuse color, a microfacet model for the surface reflection, and a bump map derived from the color maps to introduce surface normal details. However, this approach has several shortcomings.

First, wood is fundamentally a volume phenomenon (see Figure 2). Texture maps derived directly from 2D photographs serve well for flat surfaces (including wood veneer), and these are easily trans-

Permission to make digital or hard copies of part or all of this work for personal or classroom use is granted without fee provided that copies are not made or distributed for profit or commercial advantage and that copies bear this notice and the full citation on the first page. Copyrights for thirdparty components of this work must be honored. For all other uses, contact the owner/author(s). (c) 2016 Copyright held by the owner/author(s).

SA '16 Technical Papers, December 05-08, 2016, Macao ISBN: 978-1-4503-4514-9/16/12

DOI: http://dx.doi.org/10.1145/2980179.2980255 
ferable from one flat surface to another. However, curved and other more complex-shaped surfaces carved out of wood are difficult to photograph; the alternative of simply wrapping a flat texture around such a surface is often unsatisfactory. In addition to the inherent topological challenges in doing so, wood has distinctive, large-scale, volumetric structures such as growth rings whose surface patterns depend on the shape of that surface.

Furthermore, wood is composed of long, thin cells (fibers) that reflect light very anisotropically, which (with a clear smooth finish) causes a dramatic subsurface specular highlight. Previous work [Marschner et al. 2005] has shown that the surface reflectance of finished wood can be represented well by a model that includes a diffuse component and a separately colored fiber-reflection component that is controlled by a direction texture giving the 3D direction of fibers at every point on the surface.

Procedural 3D textures for color variation in wood have been demonstrated before, and very realistic wood appearance has been demonstrated using color and direction textures acquired using many images under controlled illumination, but both types of prior work have important limitations: previous procedural wood textures do not to justice to the beauty of the material, and measured textures are expensive in terms of time and equipment and cannot easily be transfered to models of different shape and size than the one measured.

This paper aims to get the best of both worlds. The first contribution is to define a $3 \mathrm{D}$ procedural texture that produces realistic finished wood with all visually important features included. We present methods to simulate growth rings, fibers, pores and rays, including their effects on both diffuse and surface and subsurface specular components of the reflectance. These features are procedural, so they naturally adapt to any geometry or sawing plane, and they are modular, in that they admit a variety of possible methods for specification, which can easily be transferred, combined, and/or replaced.

Second, we demonstrate how to achieve realistic wood figure, i.e. anisotropic secondary highlights that vary in the patterns characteristic of particular highly prized types of wood. The core challenge is defining 3-dimensional distortions of the fiber structure, which result in fiber orientation fields that closely approximate their natural counterparts. This gives rise to results seen in Figure 1.

\section{Related work}

Our method builds directly on previous work in wood appearance and texture synthesis.

Wood BRDF. The BRDF of our method is that of [Marschner et al. 2005], with minimal changes and with added importance sampling. This BRDF is the sum of a surface reflection component, a diffuse component and a subsurface, anisotropic specular component. (In this paper, by "specular" we mean glossy, not delta reflection.) For the subsurface specular component the BRDF models the reflection from subsurface fibers in much the same way reflection is modeled for hair and fur. The effect is that incoming light is scattered into a cone with some spread. Please refer to the original paper for the detailed definition and rationale behind the BRDF model.

This BRDF is able to capture effects seen in real wood that cannot be reproduced by surface-only anisotropy such as the Ward model [Ward 1992]. However, acquiring parameter data has long been a practical problem in using this wood BRDF. Marschner et al. [2005] use a specialized fitting process which requires specific equipment and a large number of photographs per sample, resulting in small amount of 2D texture. In contrast, our volumetric method

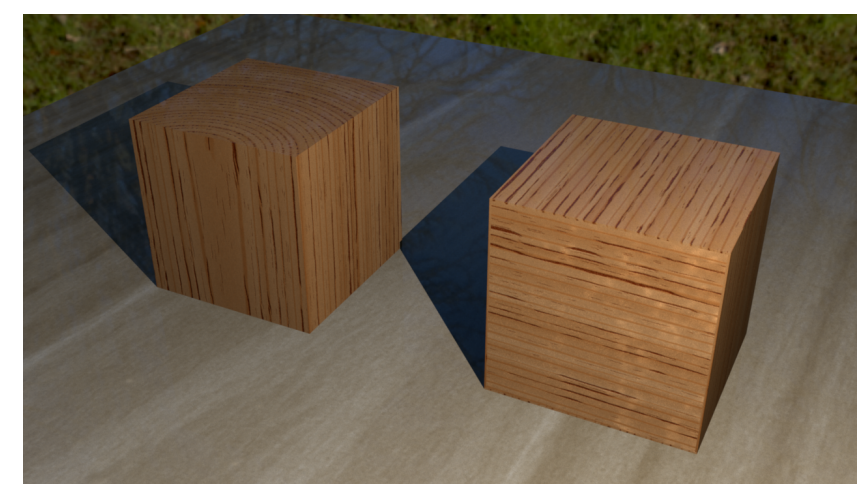

Figure 2: A rendered comparison of a carved wooden cube (left) and a veneered one (right). The carved block shows one face of each of the three major cutting planes: tangential on the left, radial on the right, and transverse on the top. The surface of the veneered block is made of six distinct thin slices and shows a tangential face on all sides. Clearly, it is substantially harder to produce the result on the left using $2 D$ texturing techniques. Public domain environment map by GiantCowFilms.
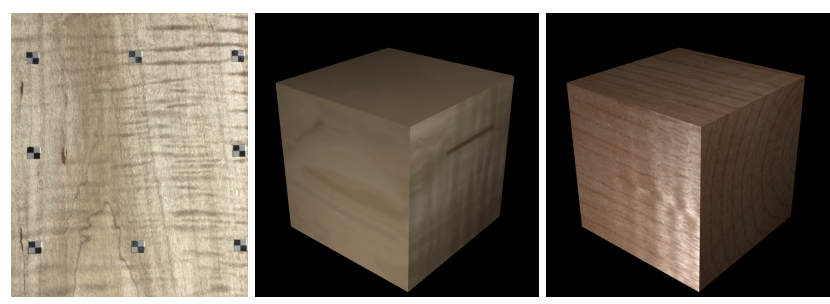

Figure 3: Generating solid texture by $2 D$-to-3D synthesis does not work well for wood. Left: Rendering produced using $2 D$ data from [Marschner et al. 2005]. Center: Rendering of the 128x128x128 solid texture set from [Kopf et al. 2007], which was synthesized from a crop of the same $2 D$ data. The results lack the global correlations found in real wood such as (approximately) cylindrical growth ring surfaces. Right: Rendering from our model, voxelized to the same texture resolution.

allows arbitrarily large synthesized texture and does not require 2dimensional UV-mapping. (Note, however, that we do not use volume rendering, but simply "carve" the parameters for the BRDF out of the volumetric model.)

Solid textures. Solid textures [Peachey 1985] are a natural fit for wood, as many features of wood, such as growth rings, are fundamentally solid phenomena. Using solid textures also obviates the need for explicit surface parameterization. However, solid textures generally have to be defined procedurally, and previous procedural models for wood, often seen as demos or examples in rendering systems, have not produced very realistic results. A 3D voxelized representation could produce higher quality, but it is not clear where the data would come from, and storing the volume naively would be prohibitively expensive.

Noise functions. A key component of most procedural textures is noise functions. A survey is given in [Lagae et al. 2010]. Excessive regularity in a texture looks unnatural and jarring, and introducing (pseudo-)randomness helps produce pleasing variation in the resulting texture. Modeling wood as a solid texture consisting of cylindrical growth rings distorted by a noise function is a wellknown practical technique [Lewis 1989; Buchanan 1998; Lefebvre and Poulin 2000]. However, previous techniques have stopped at 


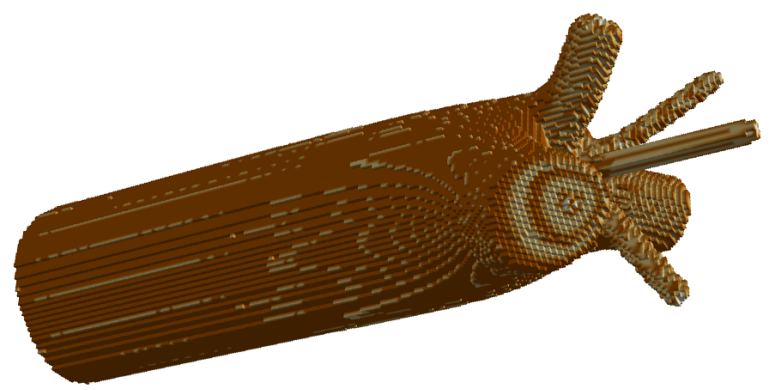

Figure 4: An example of progressive growth modeling, generated using the level set model of [Sellier et al. 2011] coupled to a phloem transport model [Sellier and Harrington 2014]. Such models are able to model large-scale topological features such as branches and knots but are comparatively computationally expensive per resolution.

approximating the diffuse color of wood growth rings.

Perhaps the most famous example of noise is Perlin noise [Perlin 1985; Perlin 2002]. Other types of noise include cellular noise [Worley 1996] and wavelet noise [Cook and DeRose 2005]. There is also the family of sparse convolution noises, which convolve a set of impulses with a kernel, first introduced by ([Lewis 1984; Lewis 1989]) and further developed by [van Wijk 1991]. More recently, the Gabor kernel has emerged as an attractive choice [Lagae et al. 2009; Lagae et al. 2011; Galerne et al. 2012] due to its excellent spatial and spectral properties. In addition to these "continuous" noise functions, it is also possible to generate a field of scattered impulses in order to place objects. It is easy to use a white noise (uniform) distribution of impulses; for our purposes we have found this to be sufficient. If less clumping is desired, one may consider blue noise distributions such as [Lagae and Dutré 2005; Lagae et al. 2008]. We have tested most of these noises and found they work well in breaking symmetry of a naive, perfect wood model. However, these generic noise functions have difficulty adequately representing the distinctive shape of various types of figure seen in real wood, such as the quilted maple shown in the teaser (Figure 1).

Exemplar-based methods. Exemplar-based methods (see [Wei et al. 2009] for a general survey) have been successful in generating a larger amount of texture from a single exemplar and are applicable to a wide variety of exemplars, though they generally struggle at reproducing large-scale coherent structures such as tree rings without some form of supervision. Works such as [Kopf et al. 2007] are even capable of generating a 3D texture from 2D exemplars. Figure 3 shows an example generated using [Kopf et al. 2007]. Unfortunately, the results do not respect the structure of the material, and the appearance is not satisfactory. Furthermore, as mentioned before, materializing a high-resolution 3D texture over a full regular Cartesian voxel grid is too expensive for many applications. We instead only ever materialize 2D textures, which we use to produce a final 3D texture procedurally. Volume effects are captured by noise and by the interaction between components rather than by full $3 \mathrm{D}$ materialization.

Progressive growth modeling. Another approach is to progressively simulate the growth of the tree over time. Examples include voxel-based approaches [Buchanan 1998], level-set methods [Sellier et al. 2011], and L-systems [Terraz et al. 2009]. Since these methods have greater access to global information, they are able to model large-scale topological features such as branches and knots as shown in Figure 4 . However, they are very computationally expensive per resolution compared to random-access methods as

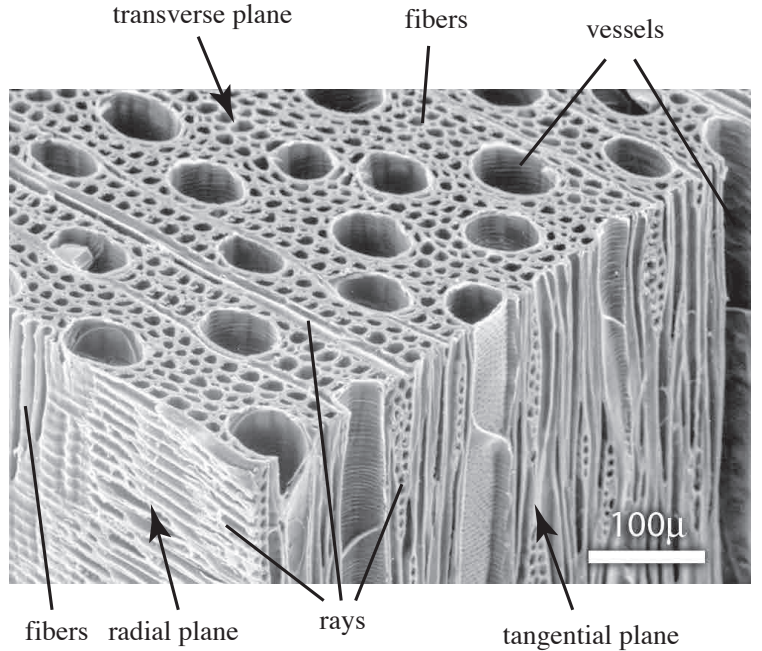

Figure 5: Scanning electron micrograph of red maple, showing vessels, rays, and fibers, as well as the radial, tangential, and transverse planes. [NC Brown Center for Ultrastructure Studies, SUNY College of Environmental Science and Forestry, Syracuse, NY]

defined in [Lefebvre 2007], which can compute the texture at any point in a constant amount of time regardless of the coordinates of the point or what parts of the texture have been previous computed. Our method is closer to the latter category, though we hope to be able to handle some of these large-scale topological features in the future.

\section{Wood anatomy background}

Our model is organized around the anatomy of wood, so we begin with some discussion of the relevant features (see Figure 6 for an illustration of the planes used to describe these features: transverse, radial and tangential). We will focus on hardwoods (trees belonging to the angiosperms; not necessarily harder wood) because they contain a greater variety of anatomical structures than softwoods and also contain nearly all woods that are prized for their appearance. Softwoods generally contain a subset of the same features and can also be handled by our model, but other woody plants used for lumber (such as bamboo) are entirely different in structure and are not within the scope of this paper [Hoadley 1980; Panshin and De Zeeuw 1970].

\subsection{Seasonal growth}

The most obvious feature in the appearance of most woods is growth rings. Growth rings result from the contrast between earlywood, which is produced during the spring, grows quickly, tends to be lighter in color, has larger and thinner-walled cells, and sometimes has larger pores (see below); versus latewood, which is produced during the summer and is the opposite. There are some species with indistinct growth rings, including many tropical hardwoods that grow in climates without strong seasonal variations.

\subsection{Longitudinal and ray fibers}

The majority of cells in wood (about $90 \%$ by volume depending on species) run in the longitudinal direction. However, there are also ribbon-like cells or clusters of cells which grow outward in the radial direction, called rays. Rays are typically very narrow in the 
170:4 - A. Liu et al.

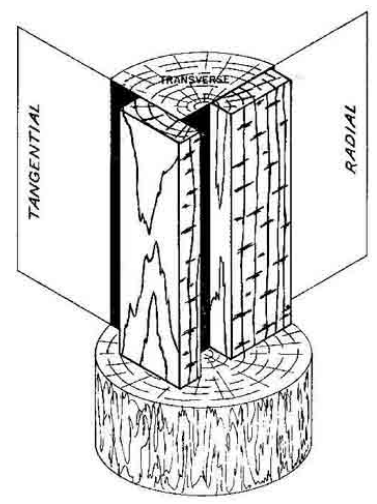

Figure 6: The principal cutting planes of wood. Diagram courtesy of Alabama Agricultural Experiment Station [Beals and Davis 1977].

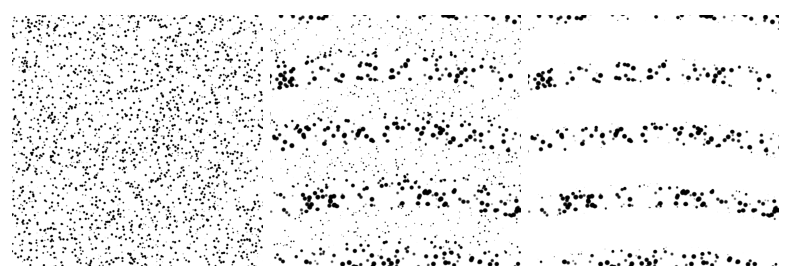

Figure 7: Vessel density may be uniform in diffuse-porous woods (left), only visible in the earlywood in ring-porous woods (right), or somewhere in-between in semi-ring-porous woods (center) as shown in these rendered diagrams.

circumferential direction and wider in the longitudinal direction. Depending on species, they may be too small to be seen easily by the naked eye or up to as large as about a millimeter thick and a few centimeters tall [Hoadley 1980].

Rays can produce a striking visual effect since their fibers run in a direction perpendicular to the main mass of fibers, thus producing a different reflective effect on the surface. On the tangential plane rays generally appear dark, whereas on transverse and radial planes they can be very bright for certain illumination configurations.

\subsection{Pores}

Pores (vessels) are hollow longitudinal tubes only found in the hardwoods. When cut, they produce openings or indentations on the surface of the wood. Pores often show up as grooves on the surface of the wood, with the length of the grooves depending on the cut. Unless the pores are filled, such indentations will remain even after the wood is coated. If a staining finish is applied to the wood, the pores will tend to absorb more of the finish, giving them a darker color than the rest of the wood.

The seasonal size and distribution of vessels depends on the species. In diffuse-porous species, the size of pores is independent of the season. At the opposite extreme are ring-porous species, where large pores occupy most of the volume of the earlywood, but the latewood pores are negligibly tiny. In these species the pores rather than the inherent color of the wood may be the primary feature of growth rings. Still other species lie between these two extremes, with larger pores in the earlywood and smaller but still significant pores in the latewood. See Figure 7 for an illustration.

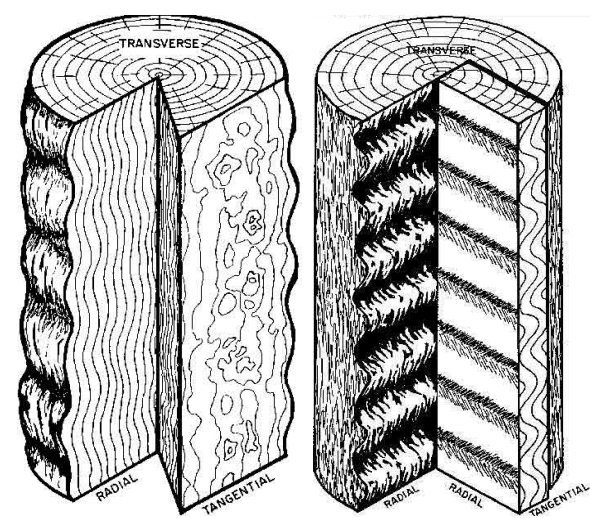

Figure 8: Left: Distortion in the radial direction produces variation in the shape of growth rings and is responsible for types of figure such as blister and quilted. Right: Distortion in the tangential direction does not affect the shape of growth rings, but is responsible for certain kinds of curly figure. Diagrams courtesy of Alabama Agricultural Experiment Station [Beals and Davis 1977].

\subsection{Figure}

The default direction of the fibers in a tree is longitudinal (parallel to the tree axis) in what is termed straight grain. Variations in the fiber direction cause distinctive visual effects known as figure. Waves in the fiber can cause deviations in the radial and/or tangential directions (see Figure 8), which is termed curly or fiddleback figure. Bumps in the radial direction may also result in blister, quilted, or birds-eye figure depending on their shape [Beals and Davis 1977]. With a clear finish, these features produce the prized secondary specular highlight patterns.

\section{Simulating wood features}

In this section, we provide an overview of all wood features modeled by our approach.

\subsection{Coordinate systems}

Below, we will assume all computation is happening in tree space: the $z$-axis of this space is aligned with the axis of the tree. We can parameterize the space using Cartesian coordinates $x, y$ and $z$, or cylindrical coordinates $r, \theta$ and $z$. In our implementation, we assume the units of tree space are centimeters; this is important to maintain correct real-world scale for all wood features. An illustration of tree space can be found in Figure 9, left. The relationship between tree space and world space can be expressed as a $4 \times 4$ affine matrix transformation for every object in the scene with the wood material.

\subsection{Basic growth rings}

A core ingredient in the color of wood at a point $\mathbf{p}$ is the time at which the point was laid down during the growth process. In the simplest case, the color can be chosen as a binary decision between specified earlywood and latewood colors. We model this as a square wave as a function of radius $r$; the widths of the rings can be user-specified. However, the earlywood-latewood transition is often fuzzy, so we allow for controllable smoothing between the high and low values. The resulting simple growth rings are shown in Figure 10 (a). 

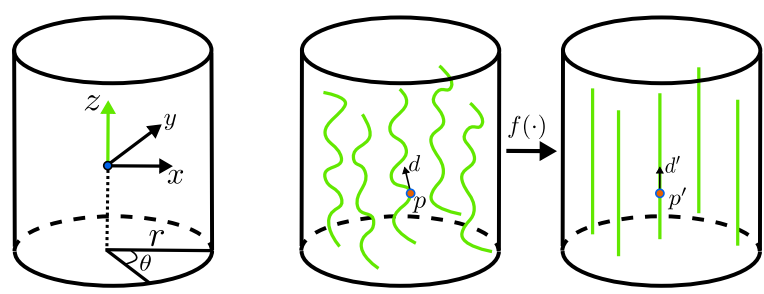

Figure 9: Left: an illustration of tree space. Right: We model distortion as a function $\mathbf{f}: \mathbb{R}^{3} \rightarrow \mathbb{R}^{3}$, mapping a position $\mathbf{p}$ in the distorted tree to a position $\mathbf{p}^{\prime}=\mathbf{f}(\mathbf{p})$ in the undistorted tree. The distorted fiber direction $\mathbf{d}$ is mapped to the undistorted direction $\mathbf{d}^{\prime}=J_{\mathbf{f}} \mathbf{d}$.

\subsection{Diffuse and specular color}

While we can specify earlywood and latewood colors independently, we hypothesize that much of the diffuse color variation in wood can be explained by varying levels of concentration of the same absorptive pigment(s). Therefore, we assume that all colors are drawn from a single Beer's law curve $c^{\alpha(\mathbf{p})}$. The base color $c$ (for example, the earlywood color) represents the color of the absorptive pigment(s), while $\alpha(\mathbf{p}) \geq 0$ is proportional to the optical depth, with a higher value representing a greater concentration of pigments.

Given a single photograph of a target wood, we can produce an estimate of the colors by taking the 25th and 75th percentiles of each channel and treating those as the earlywood and latewood colors; we can also find the exponent $\alpha$ that approximately turns earlywood into latewood color. These estimates can be further adjusted by the user if needed.

From here, $\alpha$ for any given point is determined by the sum of a few factors. First, the season of the wood determines the blend between earlywood and latewood $\alpha$. To this, we add 1D noise based on radius on the scale of years (i.e. square wave wavelength) so that the growth color varies from year to year. Then we add $3 \mathrm{D}$ noise to provide additional variation.

Secondary highlight color (i.e. the RGB weight of the fiber reflection BRDF component) is computed from the diffuse color by raising to a power less than 1 . Intuitively, this color follows the same patterns as the diffuse color, but is less saturated, because it corresponds to shorter light paths through the wood than the diffuse component. This intuition is confirmed by inspecting the measured data of Marschner et al. [2005].

\subsection{Color and ring width modulation}

Furthermore, we use a 1-dimensional Perlin noise to modulate the earlywood and latewood colors of different growth rings, and a 3D anisotropic Perlin noise, providing small-scale color detail. The modulation is achieved through modulating the power $\alpha$, as opposed to the color values directly. We also allow random variation in the growth ring sizes, by modulating with a 1-dimensional Perlin noise function of $r$. The effect of these additional modulations can be seen in Figure 10 (b).

\subsection{Distortion and fiber direction}

Real wood departs from the idealized cylindrical shape assumed above, both in terms of distinctive figure and less distinct random variations. To model these, instead of looking up points $\mathbf{p}$ directly, we apply a differentiable distortion field $\mathbf{f}: \mathbb{R}^{3} \rightarrow \mathbb{R}^{3}$ to all lookups before querying the idealized (undistorted) tree. In other words, $\mathbf{f}$ is a mapping from the distorted into the undistorted tree; this can be seen as the inverse of the intuition of building an idealized tree model and finally distorting it. This is illustrated in Figure 9, right.

To define the distortion $\mathbf{f}$, we can use a generic noise model such as Perlin noise. This works well to break the unnatural symmetry and perfection of the undistorted tree; compare Figure 10 (b) and (c). However, to achieve a natural secondary highlight, we found that Perlin noise does not give satisfying results (Figure 11), and neither do other constructions like Gabor or impulse noise. Instead, we synthesize $3 \mathrm{D}$ distortions from 2D image examples; this is the most involved part of our model, explored in the next section.

Distortion of vector fields. To distort a vector field, it is not sufficient to merely modify the lookup into an undistorted vector field $\overrightarrow{\mathbf{u}}$ by replacing $\overrightarrow{\mathbf{u}}(\mathbf{p})$ with $\overrightarrow{\mathbf{u}}(\mathbf{f}(\mathbf{p}))$. For example, merely changing the lookups into the fiber direction field does not result in any change since the undistorted fiber direction is equal to $(0,0,1)^{T}$ everywhere. Instead, we need to use the Jacobian of the distortion function. Since $\mathbf{f}$ maps the distorted tree to the undistorted tree, we observe that $J_{\mathbf{f}}$ maps the distorted vector $\overrightarrow{\mathbf{v}}(\mathbf{p})$ to the undistorted vector $\overrightarrow{\mathbf{u}}(\mathbf{f}(\mathbf{p}))$. For nonsingular $J_{\mathbf{f}}$, this can be inverted as $\overrightarrow{\mathbf{v}}=J_{\mathbf{f}}^{-1} \overrightarrow{\mathbf{u}}$.

Fiber direction. Using the above, we can find the distorted fiber direction by multiplying the inverse Jacobian $J_{\mathbf{f}}^{-1}$ by the undistorted fiber direction, which is normally $(0,0,1)^{T}$.

Jacobian computation. We found that central finite differences work well in approximating the Jacobian, while requiring 6 evaluations of $\mathbf{f}$. However, we can also compute it analytically, for distortions of the common form

$$
\mathbf{f}(\mathbf{p})=\mathbf{p}+m_{r}(\mathbf{p}) \overrightarrow{\mathbf{r}}(\mathbf{p})+m_{\theta}(\mathbf{p}) \vec{\theta}(\mathbf{p}) .
$$

Here $\overrightarrow{\mathbf{r}}$ and $\vec{\theta}$ are the normalized radial and tangential directions at $\mathbf{p}$, and $m_{r}$ and $m_{\theta}$ are scalar functions describing the magnitude of the distortion in the respective direction. (We will drop the $\mathbf{p}$ dependence for clarity from now on.) We restrict the distortion to be in the $x y$-plane, since the longitudinal direction is less interesting as it does not affect fiber directions. This allows us to design distortions that correspond qualitatively to the radial and tangential distortion seen in real wood, as in Figure 8. The Jacobian of the distortion function is then

$$
J_{\mathbf{f}}=I+\overrightarrow{\mathbf{r}} \otimes \nabla m_{r}+\vec{\theta} \otimes \nabla m_{\theta}+\left(J_{\mathbf{r}} m_{r}+J_{\theta} m_{\theta}\right)
$$

where $\otimes$ denotes an outer (tensor) product. The parenthesized terms can be ignored in practice with no obvious visual impact. This is because $\overrightarrow{\mathbf{r}}$ and $\vec{\theta}$ change slowly except close to the tree axis, so $J_{\mathbf{r}}$ and $J_{\theta}$ have small values (inversely proportional to $r$ ).

Dealing with foldover. Note that with zero distortion, $\mathbf{f}$ is the identity function, so the determinant of $J_{\mathbf{f}}$ is 1 . A conceptually subtle but visually obvious problem happens when the magnitude of the distortion is too large, and the determinant flips to negative, causing a discontinuity in distorted fiber direction. While we can make the distortion magnitudes $m_{r}$ and $m_{\theta}$ small enough that this does not happen, it is not easy to guarantee this, especially with user adjustments being allowed. If we need such a guarantee, we can compress the sum of the radial and tangential terms from equation 2 to always be less than 1 , thus modifying the equation to

$$
J_{\mathbf{f}} \approx I+\frac{\overrightarrow{\mathbf{r}} \otimes \nabla m_{r}+\vec{\theta} \otimes \nabla m_{\theta}}{\sqrt{1+\left\|\nabla m_{r}\right\|^{2}+\left\|\nabla m_{\theta}\right\|^{2}}}
$$

This ensures that $J_{\mathbf{f}}$ has positive determinant everywhere and thus never passes through a singularity. Meanwhile, small gradients are 


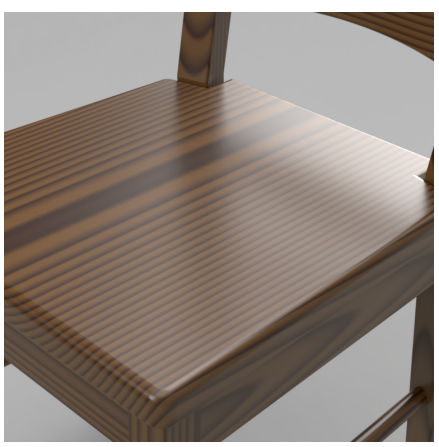

(a) basic growth rings

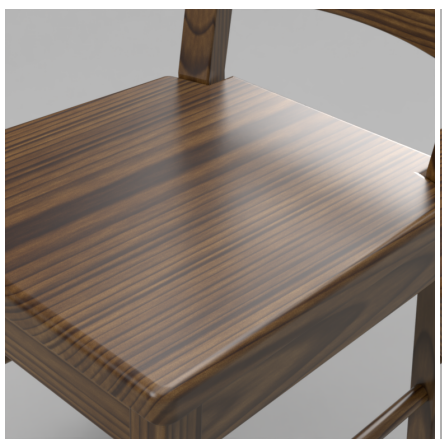

(b) color/width variations

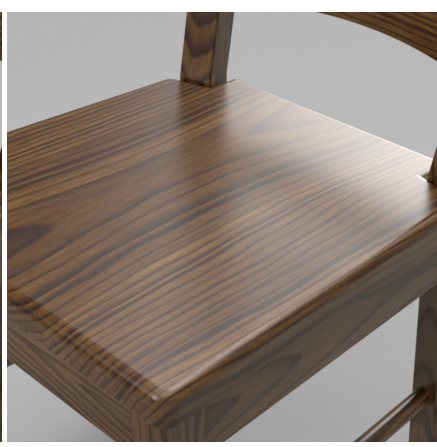

(c) distorted growth

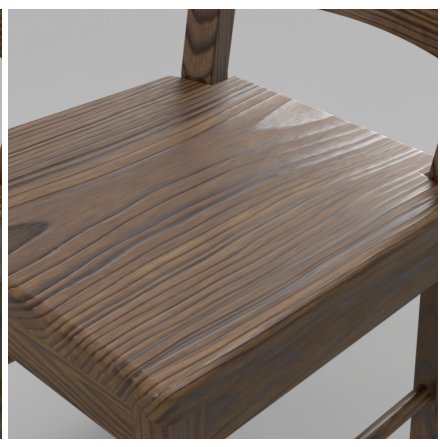

(d) pores/bump/roughness

Figure 10: Demonstrating the effect of the wood features modeled by our method.

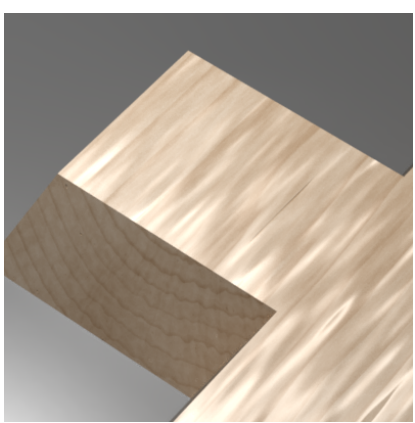

Perlin distortion

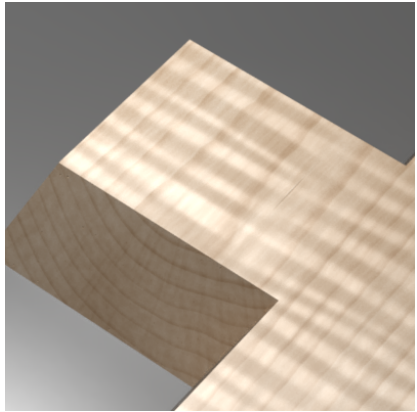

synthesized from 2D exemplars
Figure 11: Our wood model produces secondary highlights through fiber direction variations, which are determined by the wood distortion function $\mathbf{f}$. We observe that Perlin noise (left) does not lead to natural curly maple highlight appearance. We instead synthesize a more involved $3 D$ distortion from $2 D$ exemplars (right); we give more details in Section 5.

nearly unaffected. Also note that the distortion itself is not affected; just the Jacobians and the resulting fiber directions.

\subsection{Pores and rays}

We model pores (vessels) as straight lines through the undistorted tree; the distortions affect them like the rest of the wood. More precisely, we distribute the pore centers in the $2 \mathrm{D}$ cross-section of the tree using stratified random sampling with a user-defined cell size. We find the pore locations within a cell by hashing the cell index using the Burtle hash; this makes the pore locations fully procedural and alleviates the need to store them.

The effect of pores on the appearance is twofold. First, they cause surface grooves, i.e. negative offsets on the surface heightfield, which accordingly modify the shading normals. (Note, we do not currently use displacement mapping, instead just modifying surface normals according to the partial derivatives of the surface heightfield.) Second, they often cause darkening, especially if stain has been applied to the wood. We achieve both effects by defining pore weight, the influence of the pore on a given point in the undistorted tree. The weight is a decreasing function of distance $r_{p}$ from the pore. We use the Wyvill kernel $w\left(r_{p}\right)=\max \left(\left(1-r_{p}^{2} / r_{m}^{2}\right)^{3}, 0\right)$ [Shirley and Marschner 2009, p. 387], where $r_{m}$ is the distance at which the pore influence decays to zero. We then directly use this weight (scaled by a user-specified strength) to offset the heightfield and to increase the color exponent. The effect is shown in Figure
12 (b).

To represent ring-porous woods, we can vary the parameters per wood type, causing the pores to become much smaller and less dense in the latewood. This effect can be observed in both oak (Figure 12) and ash (Figure 13).

Modeling rays is similar to pores. Since rays run radially, we instead use stratified sampling in $\theta$ and $z$, but we reuse the idea of hashing and also use a Wyvill kernel to define the ray weight as a function of distance from the ray center (in Cartesian, not cylindrical space). However, here we make the weight kernel anisotropic, since we observe that rays are often not circular tubes, but resemble "ribbons" with an elliptical cross section, broader in the $z$-direction. We do not modify surface normals based on the ray weight. The effect of rays is shown in Figure 12 (c).

\subsection{Additional effects}

Below, we describe some additional simple effects, adding to the expressive power of our model.

Earlywood bump. In addition to the indentations caused by pores, in some species, earlywood is softer and absorbs more surface finish; this causes the surface heightfield to "sink" lower in earlywood regions. We model this effect by lowering the heightfield by scaling the same smoothed square wave we used to determine the colors.

Earlywood and pore roughness. We observe that the recessed surface areas (pores and earlywood rings) sometimes have higher specular roughness. We hypothesize that this is because the recessed areas are softer and absorb more polish into the wood, so less is available on the surface, or because prolonged use of a wooden object leads to less mechanical polishing in the recessed areas. This effect is easily modeled by using two user-specified roughness values for the recessed and non-recessed areas, with soft interpolation at the boundaries.

Paint and stain. We optionally allow to specify the earlywood and latewood colors manually (stain) or make them identical (paint). Figure 13 shows an example of painted ring-porous ash wood. Note how the ring effect is still important, even though the paint obscures the difference between earlywood and latewood color.

\section{Defining distortions for figure}

We have presented a decomposition of the structure and appearance of wood into simple features. Most of these features can be satisfactorily modeled using random functions, including ring width, color variation, and ray/pore placement. Random distortions 


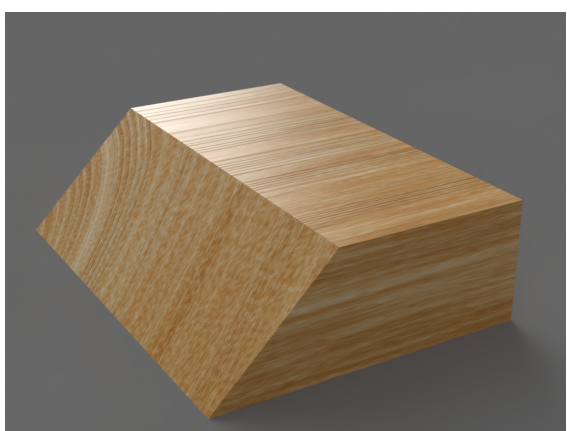

(a) no pores and rays

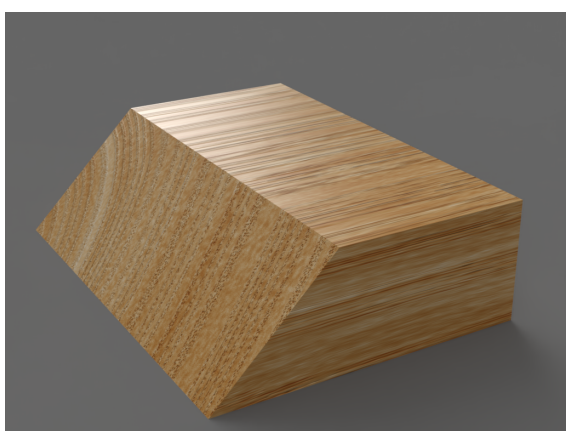

(b) with pores added

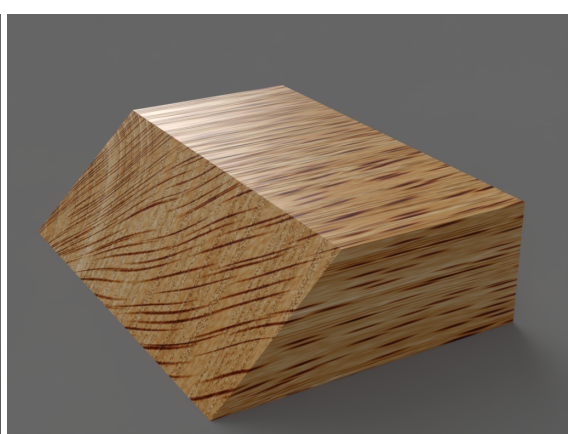

(c) with rays added

Figure 12: Illustrating the effect of pores and rays on a small block of oak.

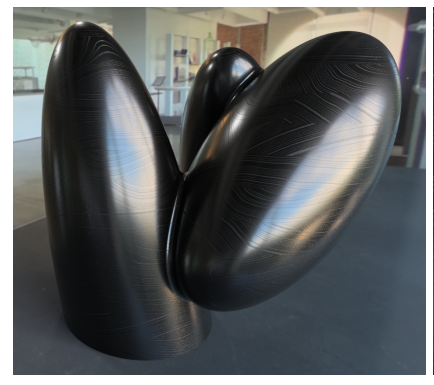

Painted ash wood sculpture rendering

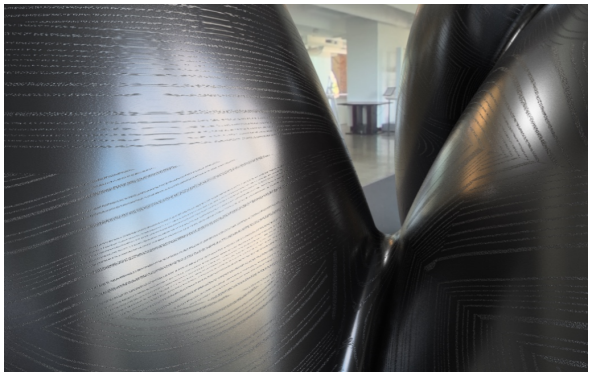

Figure 13: A sculpture made of painted ash wood, illustrating a common ring-porous appearance of this species. The paint obscures the difference between earlywood and latewood color, but the surface normal variations due to pores still reveal the growth rings. Sculpture by Wendell Castle. Photograph and model of sculpture used with permission from Carl Bass.

also suffice to simulate the minor irregularities found in wood with straight grain, but the abnormal growth patterns discussed in Section 3.4, which lead to the beautiful figure seen on finished wood surfaces, have specific structure that is not amenable to generation from noise. In this section we discuss how we author these distortions - specifically $m_{r}$ and $m_{\theta}$ in Equation 1 - which are responsible for both growth ring shapes and figured highlight patterns. (Distortions in the $z$ direction are not needed for common types of figure, so we omit $m_{z}$.)

Our approach to modeling figure starts with the observation that the patterns we need to model are generally functions of only two of the three polar coordinates. Curly or fiddleback figure, as in Figure 11, is produced by oscillations in the $\theta$ direction that vary quickly in the $z$ direction and somewhat in the $r$ direction, whereas blister, quilted, and bird's-eye figure are produced by distortions in the $r$ or $\theta$ directions that vary predominantly with $z$ and $\theta$.

We seek to provide a direct means of defining these patterns so that they look correct when sliced along the relevant plane and remain consistent throughout the volume. We do this by using a onechannel 2D texture to define the displacement magnitude in the $r$ or $\theta$ direction, i.e. $m_{r}$ or $m_{\theta}$ of Equation 1.

In the case where the distortion varies predominantly in $r$ and $z$, the texture respresents a function of $r$ and $z$. The scheme is trivial: simply revolve a one-channel $2 \mathrm{D}$ texture around the $z$-axis, and interpret the value as a displacement in either the $r$ or $\theta$ direction as shown in Figure 14.

The case where the distortion varies predominantly in $\theta$ and $z$ is more difficult : we must define a globally consistent distortion magnitude as a function of $\theta$ and $z$. Simply making distortion a function of these variables (i.e. wrapping the texture around a cylinder and projecting along the $r$ direction) leads to severe stretching of the map as $r$ changes. Our solution to this problem instead wraps the 2D texture onto an Archimedean scroll (again, shown in Figure 14) and is outlined in the following section.

Example images for the four combinations of these two cases along with displacement in the $r$ or $\theta$ direction are shown in Figure 16. We also discuss how we can generate distortion textures by texture synthesis in Sections 5.2.

\subsection{Defining distortion as a function of $z$ and $\theta$}

The goal of this section is to extend a 2D texture to a 3D texture, in such a way that slices of the 3D texture look like the input texture where the slicing plane is nearly tangential. In order to maintain the right look across a range of different positions, we wish to maintain constant linear rather than angular feature sizes as we get further from the $z$-axis. But since the circumference increases with radius, this requires increasing the number of features as $r$ increases. The texture therefore cannot be completely constant in the $r$ direction.

One solution might be to interpolate between textures defined on a series of concentric cylinders, each with the texture defined at the appropriate scale, and then interpolate between the cylinders. However, under such a scheme it may not be possible to tile an arbitrarily-sized texture around a given cylinder, and the uneven number of elements around each cylinder complicates addressing.

Instead, we elect to use an Archimedean scroll (an extruded Archimedean spiral), which has the advantage of unrolling into a single plane, greatly simplifying addressing and tiling. See Figure 17 for diagrams of the spiral and the grid, offset, and interpolation 
radial surface: fast $r, z$; slow $\theta$
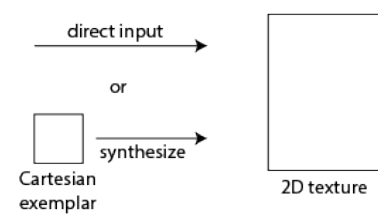

$2 \mathrm{D}$ texture

Archimedean scroll: fast $\theta, z$; slow $r$
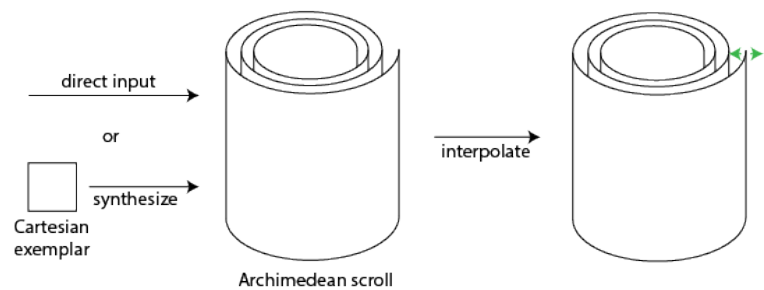

Figure 14: By assuming a cylindrical texture varies slowly along at least one cylindrical direction, we can represent it using only $2 D$ information. These $2 D$ textures can either be input directly, or synthesized using a small exemplar. The interpolation scheme for the spiral case is shown in Figure 17.
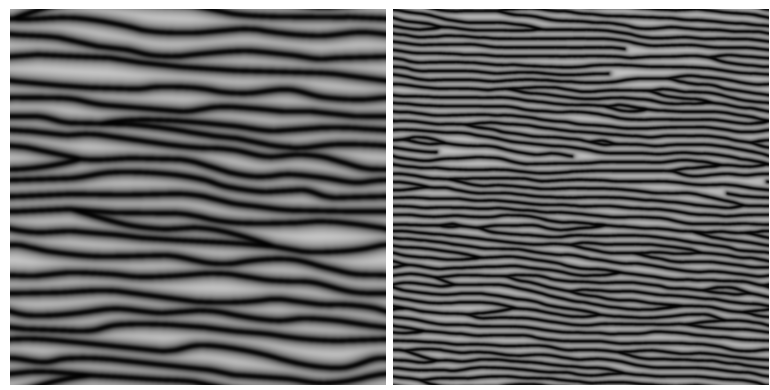

Figure 15: Given a small exemplar texture, we can use standard methods to synthesize a larger, tileable texture. This texture is then placed on either the radial plane or Archimedean scroll as per Figure 14.

scheme described in the rest of this section.

Specifically, let $t$ be the total angle of the spiral starting from the center; we parameterize the spiral by $s=\frac{t}{2 \pi}-1 \in[-1, \infty)$, the number of turns of the spiral not counting the innermost turn. Let $r_{1}$ be the spacing between turns-typically much larger than the size of a texel. The equation of the spiral is

$$
\frac{r}{r_{1}}=2 \pi t=s+1
$$

To actually index a point into the spiral, we use two parameters $n$ and $a$.

The radial parameter $n$ is equal to one less than the number of turns of the spiral crossed by a line between a the origin and a given point; $n$ is real valued and increases linearly along radii between turns. For a point $(r, \theta)$ in polar coordinates, we have

$$
n= \begin{cases}\frac{r}{r_{1}}-\frac{\theta}{2 \pi} & \text { if } \frac{r}{r_{1}} \geq \frac{\theta}{2 \pi} \\ \frac{2 \pi}{\theta} \frac{r}{r_{1}}-1 & \text { otherwise }\end{cases}
$$

where inside the innermost turn we interpolate linearly from -1 at the origin to 0 at the innermost turn of the spiral.

After computing $n$, we can determine the $s$ of the point on the spiral directly inside $(r, \theta)$ by

$$
s=\lfloor n\rfloor+\frac{\theta}{2 \pi}
$$

From here, we compute $a$, the circumferential parameter, which is also real-valued. This is equal to the area between the origin and the turn directly outside $(r, \theta)$, not counting the area $\pi / 3$ inside the innermost turn. We choose to use the area instead of arc length, as the area has a simpler expression and the difference is negligible when not near the origin.

$$
a= \begin{cases}\pi\left(s^{2}+s\right) & \text { if } s \geq 0 \\ \frac{\pi}{3}\left(s^{2}+2 s\right) & \text { otherwise }\end{cases}
$$

Texel centers lie on the spiral, but they are indexed by the area within one turn further away (see Figure 17). In practice, to avoid sharp changes at the origin, we fix all texel values for $a<0$ to be the same as $a=0$.

To interpolate a texture, we take the nearest points on the spiral on each of the nearest turns (e.g. $s$ and $s+1$ for linear interpolation), compute $a$ for each nearest point, interpolate within each turn using the fractional part of $a$, and then interpolate the resulting values between turns using the fractional part of $n$.

In addition to texturing, these can also be used to define a grid and offsets in order to produce Perlin, sparse convolution, or other forms of noise, with anisotropy naturally aligned with polar coordinates.

\subsection{Synthesis}

In addition to direct authoring of the entire texture, it is convenient to be able to create tileable textures, and to synthesize a larger texture from a smaller one. The radial surface is already a 2D Cartesian surface, and the Archimedean spiral can be unwrapped into one. Synthesis of larger and/or tileable textures can therefore done effectively for these using standard 2D-to-2D texture synthesis methods such as those given in [Lefebvre 2014]. Since the viewer typically only sees a 2D slice through the final 3D texture, and many different features participate in the final result, naive tiling is not as obvious as it would be on a 2D surface; however, if less regularity is desired, on-the-fly texture synthesis (again as given in [Lefebvre 2014]) or Wang tiling [Cohen et al. 2003] could easily be used.

\subsection{Appearance of figure}

Figures 1 and 16 show the effects of distortions on the appearance of the cut surface. Variation in the distortion shows in the color patterns and also causes changes in the fiber directions that dramatically change the appearance of the subsurface specular highlight. Growth ring shapes are affected only by distortions in the $r$ direction, since distortions in $\theta$ only move points parallel to the rings. For the fiber highlight, the most dramatic effects are caused by distortions perpendicular to the surface, since these cause the fiber directions to swing out of the plane. Distortions parallel to the surface (seen near the right side of each image in the $r$ column and near the left side of each image in the $\theta$ column) only rotate the fiber direction in the surface, producing figure that is only noticeable when the illumination is far from the normal. We used two distortion maps to produce figure in this paper, one texture (Figure 15) containing near-parallel ripples to produce curly, or fiddleback, figure and one with more irregular sharp-bottomed valleys separated by round-topped hills to produce quilted figure. 
Rendered cut

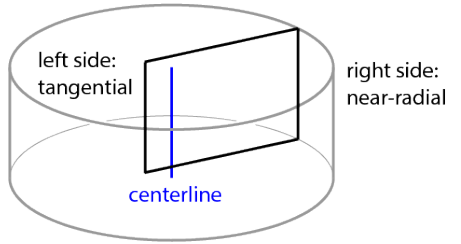

Top view

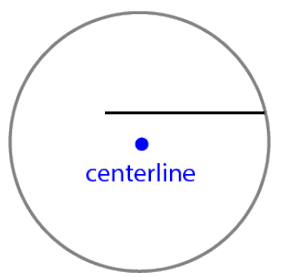

Displacement in $r$ direction

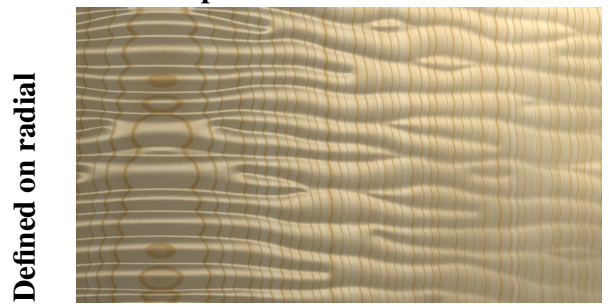

radial $\longrightarrow$

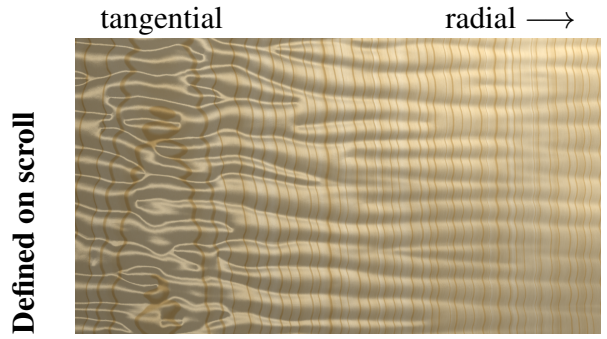

Displacement in $\theta$ direction

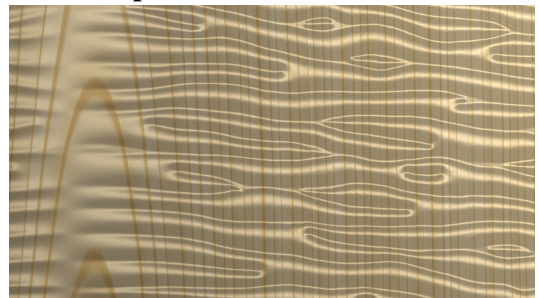

tangential

radial $\longrightarrow$

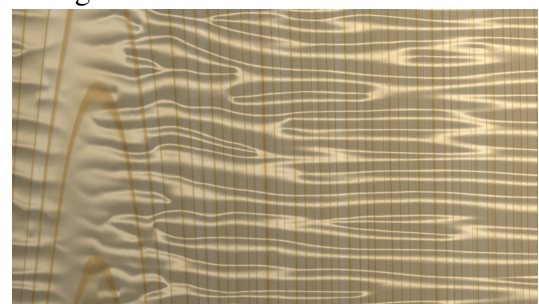

Figure 16: Example renderings of the four combinations of displacement in the $r$ versus $\theta$ directions, and placing the input texture on the radial plane versus on the Archimedean spiral. The top-right and bottom-left images correspond to the cases shown in Figure 8. The rendered cut is tangential on the left side and near-radial on the right side. Figure is exaggerated and other features are simplified for exposition. See the supplemental material for videos of the four cases.

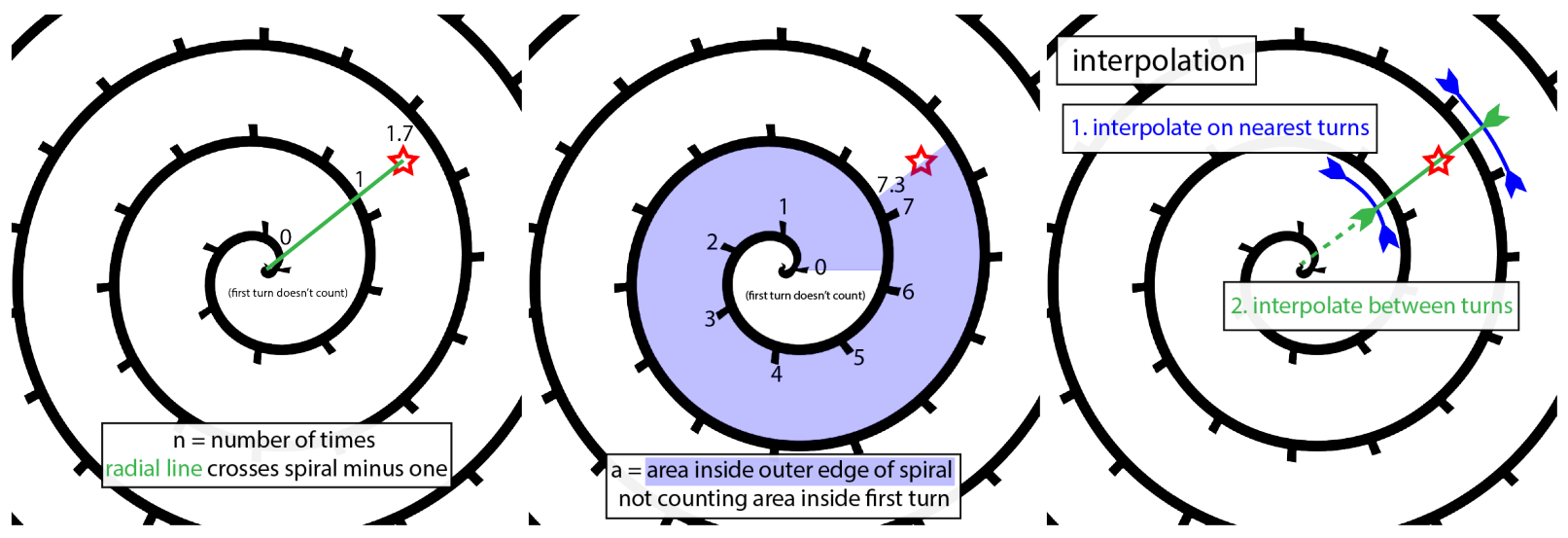

Figure 17: Archimedean spiral. Left: $n$ is the number of times a radial line crosses the spiral minus one (linearly interpolated if a point lies between turns). Center: $a$ is the area between the origin and the turn directly outside of a given point, not counting the area inside the innermost turn. Note that while texels lie on the spiral, they are indexed by the area within one turn further away. Right: Interpolation procedure. First, we interpolate within each of the nearest turns of the spiral. Then we interpolate between the results of each turn to get the value at the queried point.

\section{Additional results}

We implemented our model in the Mitsuba renderer [Jakob 2010], and also in Autodesk Fusion 360 , a commercial modeling and rendering system. To evaluate rendering performance of our method, we rendered the teaser image (Figure 1) on an Intel Xeon E5-2650 v2 machine (16 cores, 32 threads), which took 310 seconds. We then re-rendered the same image, but replacing all wood materials with a simple white plastic; this took 247 seconds. The rendering used a standard path-tracer with 512 samples per pixel and an NL-means denoising pass. The algorithm runs on-the-fly during rendering, so there is no precomputation time. After becoming familiar with the parameters, an experienced designer can create a preset in about ten minutes.

Matching renderings to photographs. An important question is how to set the parameters of our model to match physical wood samples or their photographs. We created a GUI previewer for set- ting the parameters of the wood model, shown in Figure 18. Using this tool, combined with some skill and experience, it is possible to obtain close matches between photographs and renderings of many species, as seen in Figure 19. We also show a recording of this process in a supplementary video. Automating this process is an interesting and difficult problem left as future work.

Wooden floor. One common real-world application of wood is flooring, ranging from simple parallel board patterns to elaborate parquetry. A nested-square pattern is shown in the Sponza scene in Figure 20. A board pattern can be defined by a different world-totree affine transformation for each board; we define these by randomization. 


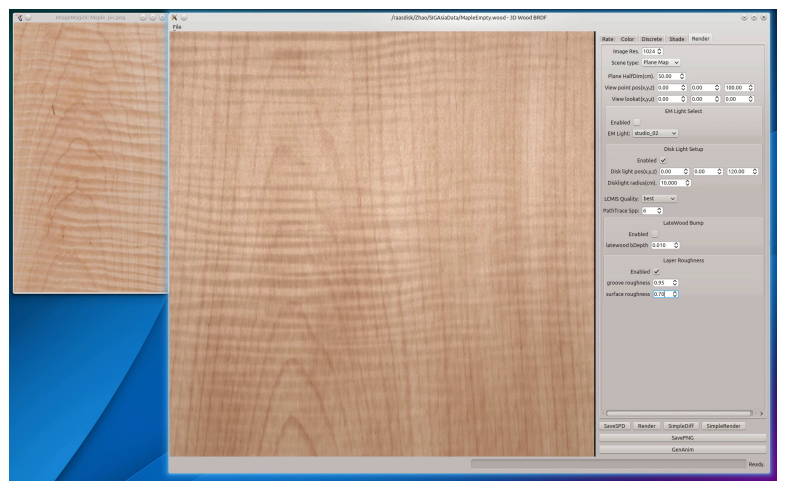

Figure 18: A screenshot of our wood previewer. On the left is a photograph of real wood, on the right is our result. We also show a recording of the parameter-setting process in a supplementary video.

\section{Conclusion and future work}

In this paper, we presented a comprehensive volumetric simulation of the appearance of wood, including components such as growth rings, pores, rays, and growth distortions. In addition, our model fully supports anisotropic specular figure caused by wood fibers, common in curly maple and other species. The fiber directions required for such secondary highlights can be derived directly from the growth distortions introduced by our model; these 3D distortions are generated from 2D exemplars using constructions designed to match the look of radial and tangential cuts. The components of our model are intuitive, easy to control, admit efficient computation and require minimal storage.

There are several possibilities for future work. We could add support for deviations from the cylindrical growth model assumed in this paper. These include knots, which are parts of small branches embedded in the trunk of the tree; burls, areas of abnormal, chaotic growth; and crotch figure, which occurs when the trunk splits into two large branches. It may also be worth deriving or approximating the wood BRDF using a more principled analysis of multiple scattering through the wood fiber geometry. Finally, a more automatic way of setting the parameters and input textures of our model from photographs would be valuable.

\section{Acknowledgements}

Funding for this work was provided by National Science Foundation grant IIS-1011919 and by a gift from Autodesk. We would also like to thank Roberto Ziche for suggesting wood model features and creating parameter presets, John Hutchinson for support and suggestions, Kate Salesin and Lydia Wang for their help in preparing and photographing the wood samples, and François Guimbretière for providing lab space for preparing the wood samples.

\section{References}

Beals, H. O., AND DaVIS, T. C. 1977. Figure in wood: an illustrated review. Tech. Rep. 486, Alabama Agricultural Experiment Station, Auburn, Alabama, USA, Jan.

BUCHANAN, J. W. 1998. Simulating wood using a voxel approach. In Computer Graphics Forum, vol. 17, Wiley Online Library, $105-112$.

ACM Trans. Graph., Vol. 35, No. 6, Article 170, Publication Date: November 2016
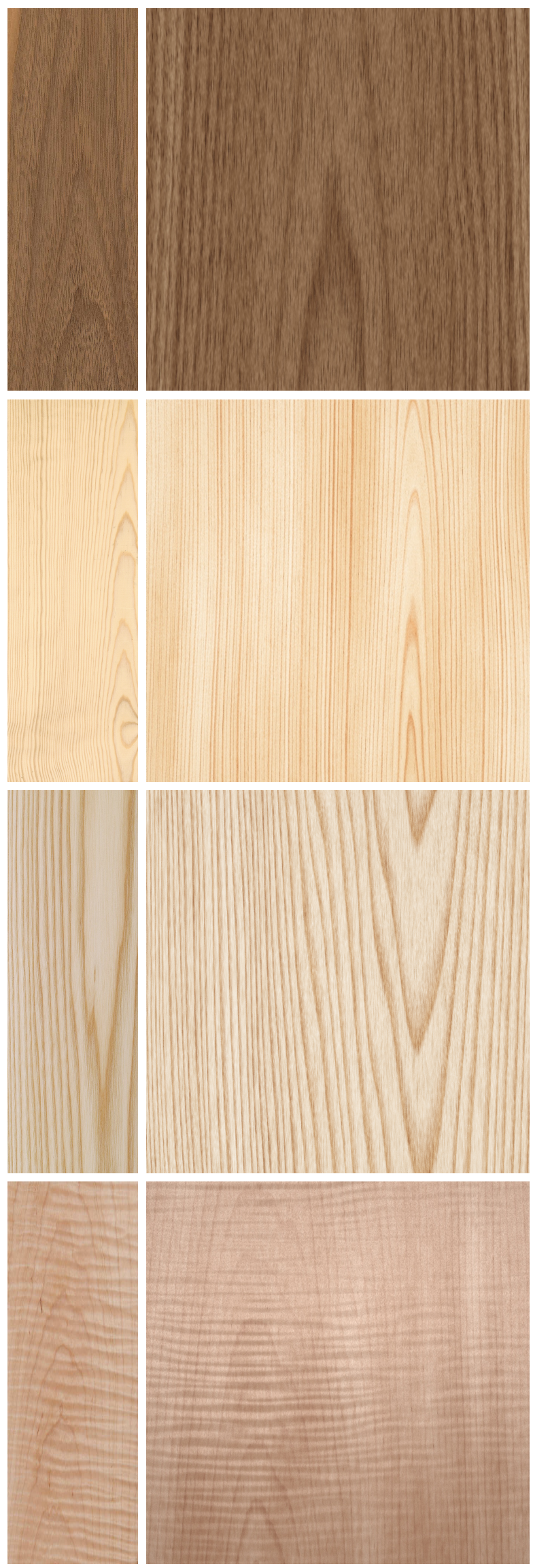

Figure 19: Photo (left) vs. rendering (right) for different wood species, top to bottom: walnut, pine, ash and curly maple. 


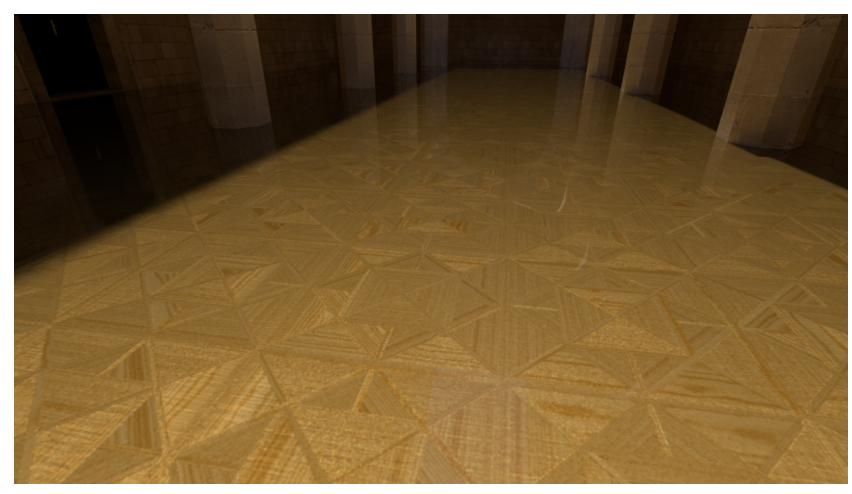

Figure 20: Nested-square parquetry floor pattern in the Sponza scene. Model by Marko Dabrovic, Kenzie Lamar, and Morgan McGuire; downloaded from Morgan McGuire's Computer Graphics Archive [McGuire 2011].

Cohen, M. F., Shade, J., Hiller, S., And Deussen, O. 2003. Wang tiles for image and texture generation. In ACM SIGGRAPH 2003 Papers, ACM, New York, NY, USA, SIGGRAPH '03, 287-294.

Cook, R. L., AND DeRose, T. 2005. Wavelet noise. In ACM SIGGRAPH 2005 Papers, ACM, New York, NY, USA, SIGGRAPH '05, 803-811.

Galerne, B., Lagae, A., Lefebvre, S., and Drettakis, G. 2012. Gabor noise by example. ACM Trans. Graph. 31, 4 (July), 73:1-73:9.

HOADLEY, R. B. 1980. Understanding wood: a craftsman's guide to wood technology. Taunton Press, Newtown, Connecticut, USA.

JАКОВ, W., 2010. Mitsuba renderer. http://www.mitsubarenderer.org.

Kopf, J., Fu, C.-W., Cohen-Or, D., Deussen, O., LischinSKI, D., AND WonG, T.-T. 2007. Solid texture synthesis from 2d exemplars. In ACM SIGGRAPH 2007 Papers, ACM, New York, NY, USA, SIGGRAPH '07.

LAGAE, A., AND DUtRÉ, P. 2005. A procedural object distribution function. ACM Trans. Graph. 24, 4 (Oct.), 1442-1461.

Lagae, A., Kaplan, C. S., Fu, C.-W., Ostromoukhov, V., AND DEUSSEN, O. 2008. Tile-based methods for interactive applications. In ACM SIGGRAPH 2008 Classes, ACM, New York, NY, USA, SIGGRAPH '08, 93:1-93:267.

Lagae, A., Lefebvre, S., Drettakis, G., and Dutré, P. 2009. Procedural noise using sparse gabor convolution. In $A C M$ SIGGRAPH 2009 Papers, ACM, New York, NY, USA, SIGGRAPH '09, 54:1-54:10.

Lagae, A., Lefebvre, S., Cook, R., DeRose, T., Drettakis, G., Ebert, D. S., Lewis, J., Perlin, K., AND ZWICKER, M. 2010. A survey of procedural noise functions. In Computer Graphics Forum, vol. 29, Wiley Online Library, 2579-2600.

Lagae, A., Lefebvre, S., AND Dutré, P. 2011. Improving gabor noise. IEEE Transactions on Visualization and Computer Graphics 17, 8, 1096-1107.

Lefebvre, L., AND Poulin, P. 2000. Analysis and synthesis of structural textures. In Graphics Interface, vol. 2000, 77-86.
Lefebvre, S. 2007. Part iv: Runtime texture synthesis. In ACM SIGGRAPH 2007 Courses, ACM, New York, NY, USA, SIGGRAPH '07.

LEFEBVRE, S. 2014. Synthése de textures par l'exemple pour les applications interactives. Habilitation thesis, Université de Lorraine.

LEWIS, J.-P. 1984. Texture synthesis for digital painting. In ACM SIGGRAPH Computer Graphics, vol. 18, ACM, 245-252.

LEWIS, J.-P. 1989. Algorithms for solid noise synthesis. ACM SIGGRAPH Computer Graphics 23, 3, 263-270.

Marschner, S. R., Westin, S. H., Arbree, A., And Moon, J. T. 2005. Measuring and modeling the appearance of finished wood. In ACM SIGGRAPH 2005 Papers, ACM, New York, NY, USA, SIGGRAPH '05, 727-734.

MCGuire, M., 2011. Computer graphics archive, August.

Panshin, A. J., And De Zeeuw, C. 1970. Textbook of wood technology, 3rd ed. McGraw-Hillm Inc., New York, New York, USA.

PEACHEY, D. R. 1985. Solid texturing of complex surfaces. In Proceedings of the 12th Annual Conference on Computer Graphics and Interactive Techniques, ACM, New York, NY, USA, SIGGRAPH '85, 279-286.

PERLIN, K. 1985. An image synthesizer. In Proceedings of the 12th Annual Conference on Computer Graphics and Interactive Techniques, ACM, New York, NY, USA, SIGGRAPH '85, 287296.

PERLIN, K. 2002. Improving noise. In Proceedings of the 29th Annual Conference on Computer Graphics and Interactive Techniques, ACM, New York, NY, USA, SIGGRAPH '02, 681-682.

Sellier, D., And Harrington, J. J. 2014. Phloem transport in trees: A generic surface model. Ecological Modelling 290, 102-109.

Sellier, D., Plank, M. J., And Harrington, J. J. 2011. A mathematical framework for modelling cambial surface evolution using a level set method. Annals of botany 108, 6, 10011011.

Shirley, P., AND Marschner, S. 2009. Fundamentals of Computer Graphics, 3rd ed. A. K. Peters, Ltd., Natick, MA, USA.

Terraz, O., Guimberteau, G., Mérillou, S., Plemenos, D., AND GHAZANFARPOUR, D. 2009. 3gmap 1-systems: an application to the modelling of wood. The Visual Computer 25, $2,165-180$.

VAN WIJK, J. J. 1991. Spot noise texture synthesis for data visualization. In Proceedings of the 18th Annual Conference on Computer Graphics and Interactive Techniques, ACM, New York, NY, USA, SIGGRAPH '91, 309-318.

WARD, G. J. 1992. Measuring and modeling anisotropic reflection. In ACM SIGGRAPH Computer Graphics, vol. 26, ACM, 265272.

Wei, L.-Y., Lefebvre, S., Kwatra, V., Turk, G., et al. 2009. State of the art in example-based texture synthesis. In Eurographics 2009, State of the Art Report, EG-STAR, 93-117.

Worley, S. 1996. A cellular texture basis function. In Proceedings of the 23rd Annual Conference on Computer Graphics and Interactive Techniques, ACM, New York, NY, USA, SIGGRAPH '96, 291-294. 